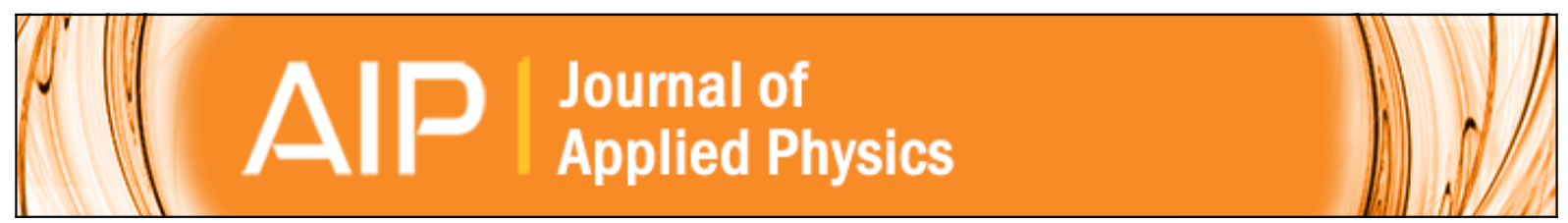

Range measurements and thermal stability study of AZ111 photoresist implanted with Bi ions

R. B. Guimarães, L. Amaral, M. Behar, F. C. Zawislak, and D. Fink

Citation: Journal of Applied Physics 63, 2502 (1988); doi: 10.1063/1.341029

View online: http://dx.doi.org/10.1063/1.341029

View Table of Contents: http://scitation.aip.org/content/aip/journal/jap/63/8?ver=pdfcov

Published by the AIP Publishing

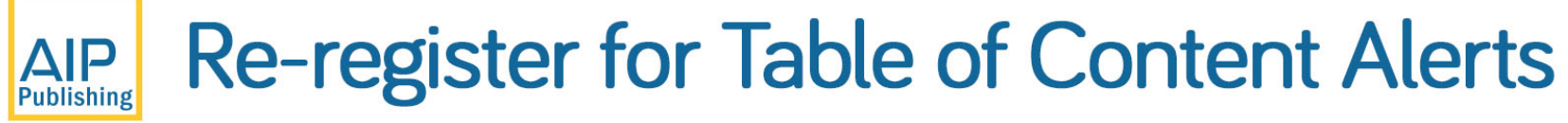

Create a profile. 


\title{
Range measurements and thermal stability study of A2111 photoresist implanted with $B$ ions
}

\author{
R. B. Guimarães, L. Amara!, M. Behar, and F. C. Zawislak \\ Instituio de Fisica, Universidade Federal do Rio Grande do Sul, 90049 Porto Alegre, Brasil \\ D. Fink \\ Hahn-Meitner-Institut, D 1000 Berlin 39, West Germany
}

(Received 5 August 1987; accepted for publication 16 November 1987)

\begin{abstract}
The Rutherford backscattering technique has been used to determine the range parameters of Bi ions implanted into AZ111 photoresist film at energies from 10 to $400 \mathrm{keV}$. An overall good agreement is found between the experimental results and the theoretical predictions by Biersack, Ziegler, and Littmark. It is also observed that a variation in the implantation dose does not affect the projected range and range straggling results, despite the fact that chemical modincation of the implanted polymer layer is detected. In addition, we find that a shallow implantation of the polymer fim with Bi ions increases the temperature at which the photoresist starts to decompose. Finally, at $300^{\circ} \mathrm{C}$ the implanted Bi atoms diffuse preferentially toward the bulk. For this temperature, two different difusion coefficients are estimated, one for the damaged region $D_{d}=1.2 \times 10^{-5} \mathrm{~cm}^{2} / \mathrm{s}$ and another for the buk $D_{b}=1.2 \times 10^{-14} \mathrm{~cm}^{2} / \mathrm{s}$.
\end{abstract}

\section{INTRODUCTION}

The investigation of ion bombardment effects on poly. mers has received increased attention during the last years. Photoresists are currently used in the semiconductor industry as masking material during ion irradiation. Ion implantation can be applied to study the effects of radiation on the solubility, the thermal stability, and the electrical conductiv. ity, as well as other physical properties of the polymers. In this framework, a considerable amount of work has been published and the results are summarized in several review papers. ${ }^{1,2}$

One of the less studied aspects of the ion implanted polymers is related to the characterization of the concentration profile of the implanted species. Despite the fact that projected ranges $\left(R_{p}\right)$ and projected range stragglings $\left(\Delta R_{p}\right)$ of implanted ions must be known to accurately determine the thickness of the masking fims used in the fabrication of microelectronic devices, very few experimental profles have been published. Several theoretical approaches can be used to calculate the ion implanted distribution parameters into poiymers. Range codes which are available and suitable for multicomponent targets are the analytic code $\mathrm{PRAL}^{3}$ and the Monte Carlo code TRIM. ${ }^{4}$ They are conveniently summarized in the recent book of Ziegler, Biersack, and Litmark (ZBL). ${ }^{5}$ The physical input into the range calculations consists of the new universal potential ${ }^{6}$ and an improved electronic stopping power, ${ }^{5}$ which is based on empirical proton stopping powers and on the concept of Brandt and $\mathrm{Ki}$ tagawa' for heavy ions.

The work described here was undertaken with several aims. First, to test the $Z B L$ predictions for complex rargets, like a photoresist where there is a general lack of experimental data. Second, to study the infuence of the implanted dose on the range parameters. It is known that high-dose implantation (typically $\phi>10^{14}$ ions $/ \mathrm{cm}^{2}$ ) can modify the chemical composition of the implanted polymers. ${ }^{1,2}$ 'Therefore, it is important to examine how a change in the implanted dose can affect the characteristic parameters of the implanted profiles. Finally, we would like to study the thermal stability of the implanted polymers. It is known ${ }^{8}$ that most of the photoresists remain stable up to $200^{\circ} \mathrm{C}$, but at higher temperatures they start to decompose losing $\mathrm{O}, \mathrm{H}$, and $\mathrm{C}$. I appears worthwile to look for possible effects of the ion implantation process on the thermal behavior of the photo. resist, in particular, for changes in the decomposition $d y$ namics as a function of temperature after implantation.

With the above purposes, we implanted ${ }^{209} \mathrm{Bi}$ into an AZ11 1 photoresist in the $10-400-k e V$ energy range. The dose effect was studied at a fixed Bi implantation energy, by changing the total fuence from $5 \times 10^{13} \mathrm{~atm} / \mathrm{cm}^{2}$ up to $8 \times 10^{14} \mathrm{~atm} / \mathrm{cm}^{2}$. Finally, we investigated the thermal behavior of the implanted polymer in the $30-400^{\circ} \mathrm{C}$ range, the results being compared with the ones corresponding to an unimplanted sample. All the depth profle measurements were performed using the Rutherford backscattering (RBS) technique.

\section{EXPERIKENTAL PROCEDURE AND DATA AAALYSIS}

Clean silicon wafers were spin coated with a AZ111 photoresist of thickness $1 \mu \mathrm{m}$ and then baked for $1 \mathrm{~h}$ at $170^{\circ} \mathrm{C}$. Small pieces of the wafers $\left(\cong 2 \mathrm{~cm}^{2}\right.$ ) were subsequently implanted with fuences and energies ranging from $5 \times 10^{14} \mathrm{~atm} / \mathrm{cm}^{2}$ at $10 \mathrm{keV}$ up to $2 \times 10^{15} \mathrm{~atm} / \mathrm{cm}^{2}$ at 400 $\mathrm{keV}$. For the study of the dependence of the range parameters on the implanted dose, five samples were implanted at an energy of $100 \mathrm{keV}$ with doses ranging from $5 \times 10^{13} \mathrm{~atm} /$ $\mathrm{cm}^{2}$ up to $8 \times 10^{14} \mathrm{~atm} / \mathrm{cm}^{2}$. All the implantations were done at room temperature in the $400-\mathrm{keV}$ ion implanter of the Institute of Physics, Porto Alegre. The beam current densities were $\cong 50 \mathrm{nA} \mathrm{cm} \mathrm{cm}^{-2}$ in order to avoid excessive heating of the samples.

Depth profiles were obtained via RBS analysis using 
$760-800-\mathrm{keV}$ alpha particles from the same implanter. Each sample was measured twice with the beam impinging per. pendicularly onto the sample's surface and under angles of $60^{\circ}-70^{\circ}$ with the sample's normal. The tilted geometry improves the depth resolution of the measurements. Backscattered alpha particles were registered by a silicon surfacebarrier detector placed at $160^{\circ}$ with respect to the beam direction. The detection resolution of the system was better than $14 \mathrm{keV}$. The beam spot on the sample was changed whenever the $\alpha$ dose reached the value of $2 \times 10^{13}$ ions $/ \mathrm{cm}^{2}$ to avoid compaction effects and formation of carbon-rich regions as a consequence of large dose irradiation with the alpha beam. On the average, the total $\alpha$ dose used to analyze each sample was around $2 \times 10^{14} \mathrm{~atm} / \mathrm{cm}^{2}$.

Data analysis was performed calculating directly from the measured spectra the four moments $\left[R_{p}, \Delta R_{p}, \gamma\right.$ (skewness), and $\beta$ (kurtosis) ] of the ion distribution. In all cases, the implanted profiles are Gaussian distributions with $\gamma \cong 0$ and $\beta \cong 3$. The values of $R_{p}$ and $\Delta R_{p}$ have been determined by using the surface approximation with the $\alpha$ particle stopping power taken from Ref. 5 . Range stragglings have been calculated after deconvolution under the usual assumptions that the estimated energy straggling of the ${ }^{4} \mathrm{He}$ ions into the AZ111 photoresist and the system resolution are both Gaus. sians. The main errors in the evaluation of the implanted profile are basically due to the uncertainty in the reported stopping powers which are around $5 \%$ (see, for example, Ref. 10), the instability of the electronic system and the lack of knowledge of the exact chemical composition of the photoresist.

\section{RESULTS}

\section{A. Dose}

In order to see whether the profle parameters are dose dependent, we have implanted four A2111 samples with total doses of $5 \times 10^{13}, 10^{14}, 5 \times 10^{14}$, and $8 \times 10^{14} \mathrm{Bi} / \mathrm{cm}^{2}$, all at a fixed energy of $100 \mathrm{keV}$. Despite this wide range of the implanted dose, the Bi profles remain basically the same. That is, they are Gaussian for all the samples, and the projected ranges and range stragglings are identical within the experimental errors, as is shown in Table 1 . This result is very important because for the lower implantation dose $\phi \leqslant 10^{14}$ bons $/ \mathrm{cm}^{2}$, no significant modifications in the chemical composition of the photoresist film has been detected as revealed by the corresponding RBS spectrum [see Fig. 1(a) ]. On the contrary, for a dose of $5 \times 10^{14}$ ions $/ \mathrm{cm}^{2}$, we

TABLE I. Dose dependence of the range parameters of $100 \mathrm{keV}{ }^{209} \mathrm{Bi} \mathrm{im}$ planted into $1-\mu \mathrm{m}$ fim of AZ111. The estimated oxygen loss as a consequence of the implantation process, is also shown.

\begin{tabular}{cccc}
\hline $\begin{array}{c}\text { Dose } \\
\left(10^{14} \mathrm{~cm}^{-2}\right)\end{array}$ & $\begin{array}{c}R_{R^{\prime}} \\
(\mathrm{A})\end{array}$ & $\begin{array}{c}\Delta R_{p} \\
(\mathrm{~A})\end{array}$ & $\begin{array}{c}\text { Oxygen loss } \\
(\%)\end{array}$ \\
\hline 0.5 & 750 & 150 & $\cdots$ \\
1 & 780 & 155 & $\cdots$ \\
5 & 760 & 160 & 20 \\
8 & 730 & 150 & 35 \\
\hline
\end{tabular}
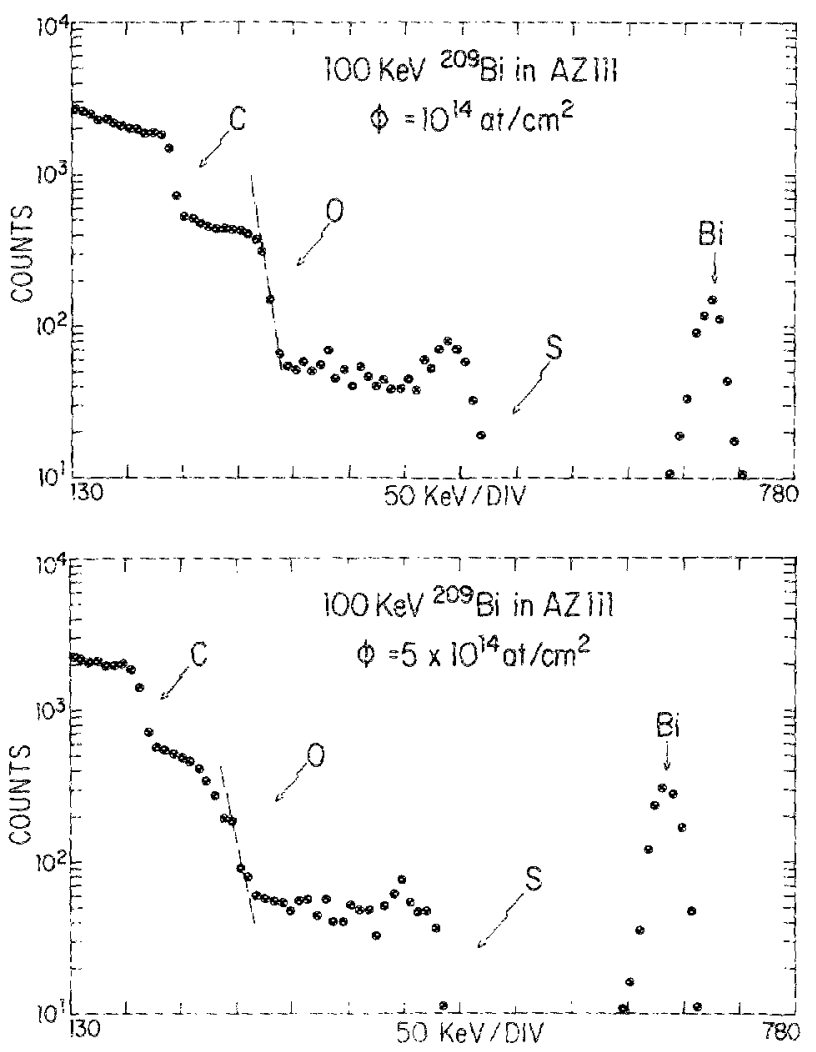

FIG. 1. (a) RBS specirum of $100 \mathrm{keV}$ Bi implanted into the AZII 1 photoresist with a dose of $10^{14}$ ions $/ \mathrm{cm}^{2}$. No significant loss of oxygen and/or carbon is observed. (b) RBS spectrum of $100-\mathrm{keV}$ Bi implanted into the AZ111 photoresist with a dose of $5 \times 10^{14}$ ions $/ \mathrm{cm}^{2}$. There is a loss of $20 \%$ of oxygen in the region from the surface isp to $1300 \AA$

have detected $20 \%$ of oxygen loss in the region from the surface up to $1300 \AA$ as is shown in Fig. $1(b)$. The loss of oxygen increases for the larger implantation dose (see Table 1). Nevertheless, the range parameters are rather insensitive to these chemical modifications.

In addition, for up to a total dose of $4 \times 10^{14} \mathrm{He} / \mathrm{cm}^{2}$, the $\alpha$ beam probe did not affect either the chemical composition of the AZ11 film or the range parameters of the implanted depth profiles. We have obtained the same results accumulating the $\alpha$ beam dose in a single spot or frequently changing the position of the spot on the target. Despite this last result, we have analyzed our samples moving the position of the $\alpha$ beam on the target, in order to avoid excessive local heating of the sample.

\section{Range measurements}

One of the major aims of the present work is to compare the experimental range parameters of $\mathrm{Bi}$ implanted into the AZ111 photoresist with the predictions by the ZBL theory. ${ }^{5}$

The universal potential and the improved electronic stopping power have been used as input to Monte Carlo simulation TRIM code program ${ }^{4}$ (1986 version) in order to calculate the characteristic parameters of the ion distributions. The TRIM calculations further allow us to determine the higher moments of the range distributions. In the present case we have obtained $\gamma=0.06-0.23$ and $\beta=2.95$ which, 
TABLE II. Experimental and TRiM calculated projected ranges $\left(R_{p}\right)$ and range stragglings $\left(\Delta R_{p}\right)$ for ${ }^{209} \mathrm{Bi}$ implanted into the $A Z 111$ photoresist film.

\begin{tabular}{rcccc}
\hline $\begin{array}{c}\text { Energy } \\
(\mathrm{keV})\end{array}$ & $R_{p}\left(\begin{array}{c}(\mathrm{exp}) \\
(\AA)\end{array}\right.$ & $\begin{array}{c}\Delta R_{p}(\exp ) \\
(\AA)\end{array}$ & $\begin{array}{c}R_{p}(\mathrm{TRIM}) \\
(\AA)\end{array}$ & $\begin{array}{c}\Delta R_{p}(\mathrm{RRIR}) \\
(\AA)\end{array}$ \\
\hline 10 & 180 & 31 & 160 & 23 \\
20 & 240 & 36 & 240 & 34 \\
30 & 310 & 50 & 300 & 42 \\
50 & 450 & 72 & 400 & 58 \\
70 & 550 & 100 & 520 & 70 \\
100 & 750 & 140 & 650 & 80 \\
200 & 1200 & 260 & 1020 & 150 \\
400 & 2200 & 450 & 1780 & 260 \\
\hline
\end{tabular}

after convolution with the experimental depth resolution function, give values of $\gamma=0.01$ to 0.1 and $\beta \cong 2.9$ which are in quite good agreement with the results obtained in the present experiment $(\gamma \approx 0 ; \beta \approx 3)$.

Table $I$ shows the $R_{p}$ and $\Delta R_{p}$ experimental values as a function of the implanted energy together with the TRIM predictions. These results are also displayed in Fig. 2, where the continuous line shows the theoretical predictions as provided by the TRIM program. For energies up to $100 \mathrm{keV}$ there is an overall good agreement (better than $8 \%$ ) between the experimental and theoretical $R_{p}$ values. However, at higher energies, the measured $R_{p}$ are systematically higher than the theoretical ones, the difference being of the order of $25 \%$ at $400 \mathrm{keV}$. Regarding the $\Delta R_{p}$ values we see the same behavior: There is good agreement between the predicted and measured values for low energy $(E<70 \mathrm{keV})$ and increasing deviation at higher energies.

In our experimental range evaluation, as well as for the theoretical TRIM code calculations, we have used the density of the AZ111 films to be $1.2 \mathrm{~g} / \mathrm{cm}^{3}$ as quoted by the supplier. " Also, for calculation purposes we have taken the nominal composition of the $\mathrm{AZ} 111$ photoresist to be $\mathrm{C}_{5} \mathrm{H}_{8} \mathrm{O}_{2}$. It is important to mention that the relative difference between the theoretical and experimental $R_{p}$ and $\Delta R_{p}$ values is independent of the assumed photoresist density.

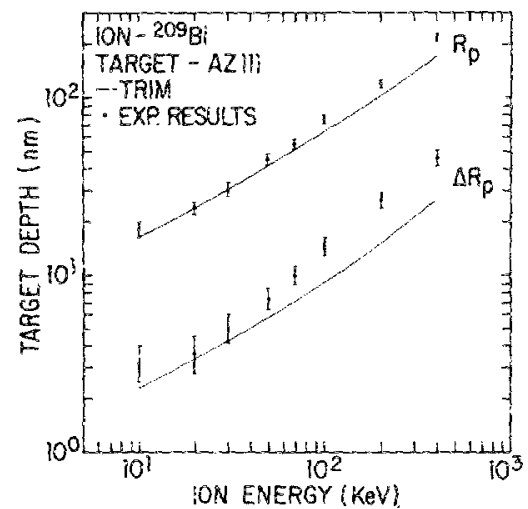

FIG. 2. Comparison of experinental and calculated projected range $R_{p}$ and range straggling $\Delta R_{p}$ as a function of energy. The points represent the experimental results. The full hines correspond to the theoretical TRMM predictions.

\section{Thermal stability}

To investigate the influence of the ion implantation process on the thermal stability of the photoresist, we have performed isochronal annealings on both unimplanted and $\mathrm{Bi}$ $50-\mathrm{keV}$ implanted samples, at $100,200,250,300$, and $350^{\circ} \mathrm{C}$ for 20 min each in a vacuum better than $10^{-6}$ Torr.

The RBS spectra of the unimplanted sample show that up to $200^{\circ} \mathrm{C}$, there is no significant change in the chemical composition of the photoresist. However, at higher temperatures the situation changes drastically as illustrated in Fig. 3. This figure shows the RBS spectra corresponding to the unannealed, annealed at 250 and $350^{\circ} \mathrm{C}$ AZ111 polymer fim. At $250^{\circ} \mathrm{C}$ the RBS spectrum shows that there is a considerable and uniform loss of oxygen (around $30 \%$ ), in all the observed depth from the surface up to $3000 \AA$. In addition, a small decrease in the $C$ amount $(\leqslant 15 \%)$ is also detected. At a higher ternperature there is an increasing loss of material and finally at $350^{\circ} \mathrm{C}$, three main features can be observed: (i) the oxygen loss is around $50 \%$; (ii) the $C$ content of the sample is reduced in $25 \%$ and; (iii) the Si edge of the substrate appears in the RBS spectrum indicating that the film has become thinner (the thickness of the film at $350{ }^{\circ} \mathrm{C}$ is $\approx 7500 \AA$ ) as a consequence of the considerabie loss of material.

On the other hand, the most important features of the thermal behavior of the implanted sample are shown in Fig. 4. Annealings up to $250^{\circ} \mathrm{C}$ did not significantly change the photoresist composition nor the Bi depth profiles. Annealing at $300^{\circ} \mathrm{C}$ results in the thermal difusion of $\mathrm{Bi}$, but still no major changes are detected in the AZ111 composition. Only at $350^{\circ} \mathrm{C}$ does the RBS spectrum of Fig. 4 reveal an appreciable loss of oxygen and a drastic diffusion of the implanted $\mathrm{Bi}$. Finally, at $400^{\circ} \mathrm{C}$ the photoresist decomposes completely (not shown in the figure).

Furthermore, we studied the diffusion behavior of the implanted Bi into the AZ111 photoresist. Analysis of ther-

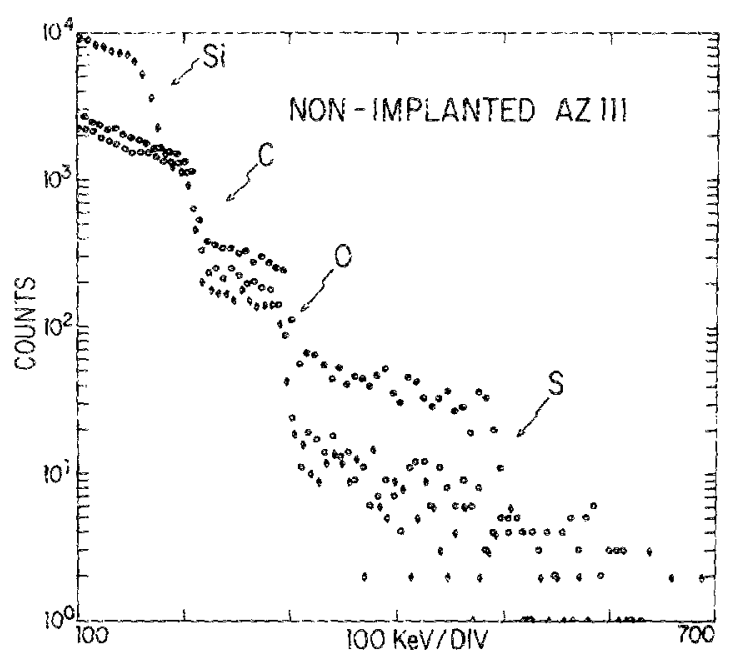

FG. 3. RBS spectra corresponding to an unimplanted AZ111 poiymer fitm: as received (full points); after $250^{\circ} \mathrm{C}$ annealing (open points) and after $350^{\circ} \mathrm{C}$ annealing (lozenge). After the last annealing there is an appreciable loss of material and as a consequence the fim thickness is reduced in $25 \%$, can be deduced from the appearance of the Si substrate edge. 


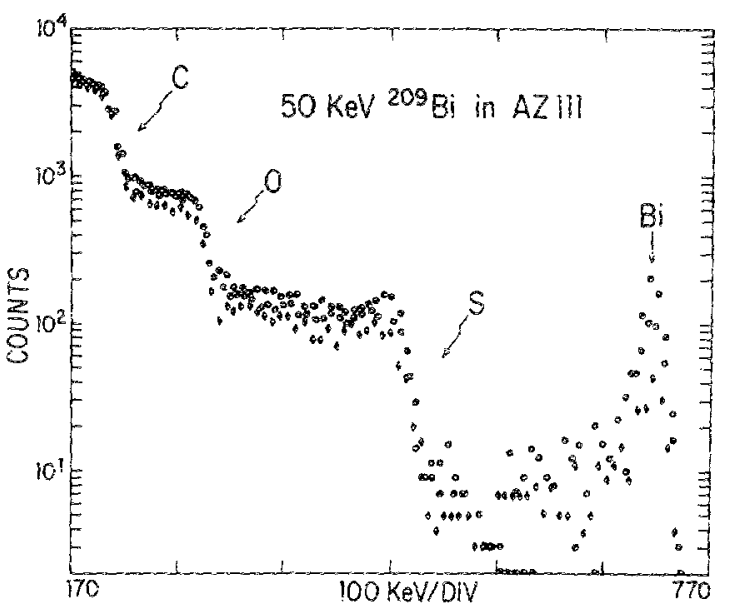

FIG. 4. RBS spectra corresponding to a shallow $10^{14} \mathrm{Bi} / \mathrm{cm}^{2}$ implartation into the polymer film: as-implanted (full points $)$; after $300^{\circ} \mathrm{C}$ annealing (open points) and after $350^{\circ} \mathrm{C}$ annealing (lozenge).

mal difusion experiments in implanted systems are difficult, essentially due to the damage produced by the implantation process, which does not always anneal out during or after the implantation, and affects the diffusion process here. To overcome these problems we analyzed our data with the numerical simulation method of "finite differences." 12 Earlier applications are given in Refs. 13 and 14. This technique calculates the diffusional change of any profile, channel by channel, and finally adds up all modified spectrum values. The diffusionai change can be different for any channel, i.e., diffusion with depth dependent diffusion coefficients $D(x)$ can be simulated. Furthermore, surface boundary effects as rapping and evaporation through the surface can be taken into account. Finally, it is possible to include trapping and detrapping probabilities $A(x)$ and $B(x)$ of the difusing particle species at each channel. Alternatively, in case of nonzero detrapping probability, the hindered diffusion can be described by an effective diffusion constant

$$
D_{\text {eff }}(x)=D(x) /[1+A(x) / B(x)],
$$

which makes the theoretical simulation process easier and more reliable, as the number of unknown variables is reduced. Therefore, this last procedure is applied here. For convenience, we separate $D(x)$ into a depth-independent value $D^{*}$ and a normalized, dimensionless depth-dependent function $f(x)=D(x)_{\text {eff }} / D *$.

Figure 5 shows the application of the above procedure. The full points represent the depth profile of $\mathrm{Bi}$ after $200^{\circ} \mathrm{C}$ annealing (which is similar to the as-implanted one). The open points show the ion distribution after annealing at $300^{\circ} \mathrm{C}$. The insert in Fig. 5 displays the depth-dependent difusion parameter used by the program in order to repro. duce the final ion distribution profile.

According to TRIM calculations, which predict that the damaged region should extend from the surface down to around $400 \AA$ depth, we determined $f(x)$ so that it is equal to 0.02 from surface to $400 \AA$ depth, then increased linearly up to 0.22 at a depth of $700 \AA$, and maintains a constant value below. This procedure simulates a high trapping probability in the damaged region, and a very low one in the undarnaged deeper zone, with a smooth transition between them. Furthermore, we assumed that the Bi could leave the photoresist and evaporate.

With these conditions, starting from the initial profile, after several iterations the program provides the depth profle shown in Fig. 5 as a full line. The agreement between the experimental result and the calculated one is quite satisfactory in particular, for the near surface (up to $450 \AA$ ) and bulk regions. The extracted diffusion coefficients for $\mathrm{Bi}$ the damaged and bulk regions of the photoresist at $300^{\circ} \mathrm{C}$ are, respectively, $D_{d}=1.2 \times 10^{-15} \mathrm{~cm}^{2} / \mathrm{s}$ and $D_{b}$ $=1.2 \times 10^{-14} \mathrm{~cm}^{2} / \mathrm{s}$.

\section{W. OISCUSSION AND CONCLUSIONS}

It is well known that energetic ions damage and modify organic materials in a chemically irreversibie fashion. Due to those radiation damage phenomena until recently, ion beam techniques have been largely overlooked in studying the properties of polymers and polymer structures. On the other hand, non-nuclear techniques give only very indirect depth profile information explaining the sparse data in the literature.

In this work we have shown conclusively, that range parameters describing implanted depth profiles of polymer layers are rather insensitive to the chemical modifications

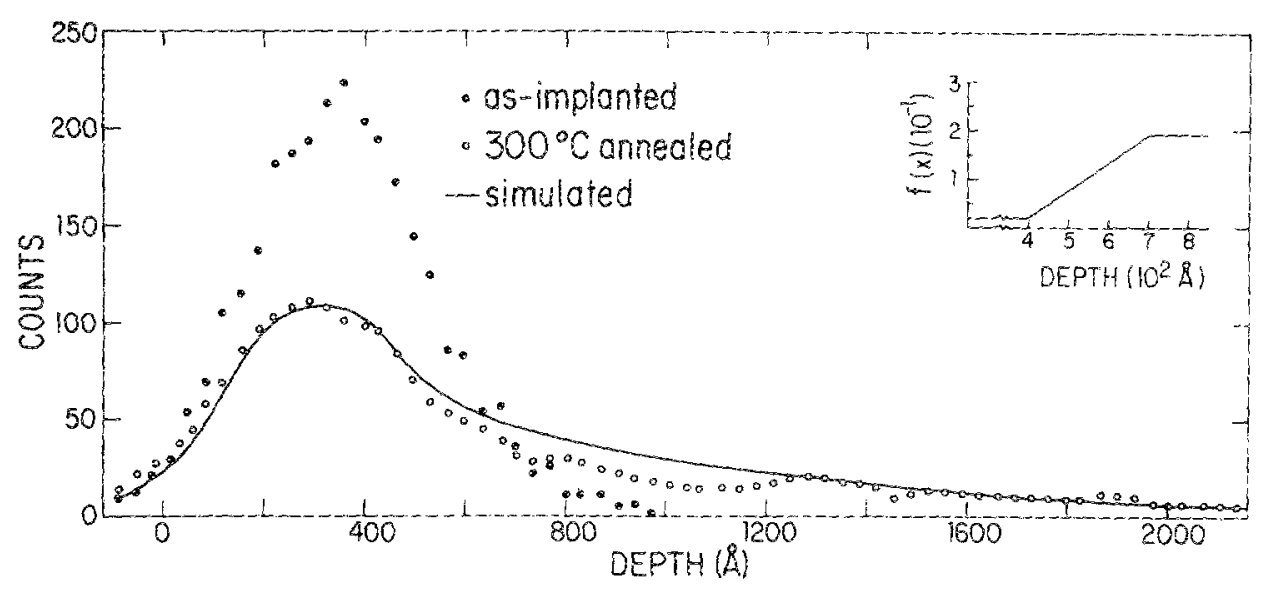

FIG. 5. Full points represent the Bi depth profle after $200^{\circ} \mathrm{C}$ annealing. The open points show the ion distribution after annealing at $300^{\circ} \mathrm{C}$. The insert displays the depth dependent diffusion parameter used in the calculation. Smooth curve represents the simulated depth profile. 
produced by the implantation process, despite the rather wide range of implanted doses. We have also demonstrated that the energetic $\alpha$ probe beam neither significantly alters the chemical composition of the AZ111 fim (in a dose range compatible with the RBS profile measurements), nor affects the range parameters of the concentration profles.

Concerning the range predictions of the ZBL theory, we can conclude that there is an overall good agreement between the calculated and experimental values. The discrepancies observed at higher energies (around $20 \%$ for $R_{p}$ and $70 \%$ for $\Delta R_{p}$ ) are beyond the experimental errors. The disagreement cannot be attributed to the lack of precise knowledge of the polymer's density, because the relative difference between the experimental and theoretical values is independent of the assumed density. On the other hand, for both the experimental and theoretical calculations we have taken the nominal composition of the polymer to be $\mathrm{C}_{5} \mathrm{H}_{8} \mathrm{O}_{2}$. If the composition is different, variations in both experimental and theoretical range values are expected, which would be a source of the observed disagreement. We feel that further experimental work is needed in order to observe if the present discrepancies are characteristic of the Bi-AZ111 system, or if some more general features are present, for example, the validity of the Bragg's rule, used for the evaluation of the experimental results, or the precision in the $\mathrm{ZBL}$ prediction for complex targets.

Concerning the thermal behavior of the implanted polymers, our results have clearly shown that as a consequence of the implantation process there is an increase in the temperature at which the photoresist starts to decompose. We have observed that the unimplanted sample starts to loose oxygen at around $200^{\circ} \mathrm{C}$, confirming previous experiments [see, for example, Ref. 81. On the other hand a low-dose and shallow implantation of Bi raises the decomposition temperature of the AZ111 polymer to $350^{\circ} \mathrm{C}$. A possible and very speculative explanation can be related to the fact that the Bi implantation creates damage and therefore inhibits the difiusion process of the oxygen. This hypothesis is, in a way, confirmed by our thermal diffusion studies of the implanted Bi. As was mentioned above, we were able to estimate two diffusion coefficients, one for the damaged region and another for the bulk. They differ by one order of magnitude which may be attributed to the retarded diffusion which occurs due to damage produced by the implantation process. Therefore, one might conclude that both processes, the rising of the decomposition temperature of the polymer and the inhibited diffusion in the implanted region could be related to the same origin, the damage produced by the implantation process.

\section{ACKNOWLEDGMENT}

We would like to acknowledge the helpiul suggestions given by Dr. J. P. Biersack.

'T. Venkatesan, Nucl, Instrum. Methods $\$ 7 / 8,461$ (1985).

${ }^{2}$ W. L. Brown, Radiat. Eff. 99, 281 (1987).

${ }^{3}$ J. P. Biersack, Z. Phys. A 39595 (1982).

J. P. Biersack and L. G. Haggmark, Nucl. Instrum. Methods 174, 257 (1980).

5. P. Biersack and U. Litrmark, in Stopping and Ranges of Ions in Matter, edited by J. F. Ziegler (Pergamon, New York, 1985), Vol. 1, p. 1.

${ }^{6}$ J. P. Biersack and J. F. Ziegler, in Ion Implaniation Techniques, Vol. 10 of Springer Series in Electrophysics, edited by H. Ryssel and H. Glawischrigg (Springer, Berlin, 1982), pp. 122-156; Nucl. Instrum. Methods 194, 93 (1982).

7W. Brand and M. Kitagawa, Phys. Rev. B 25, 5631 (1982).

${ }^{8}$ Y. Okuyama, T. Hasinimoto, and T. Yoguchi, J. Electrochem. Soc. 125, 1293 (1978).

${ }^{9}$ W. Chu, J. W. Mayer, and M.A. Nicolet, Backscattering Spectroscopy (Academic, New York, 1978).

${ }^{10}$ D. C. Sentry and R. D. Wemer, Nucl. Instrum. Methods 178, 523 (1980).

"Shypley Co., Zürich, Switzerland.

${ }^{12}$ G. D. Smith, Numerical Solution of Partial Differential Equations: Finite Difference Methods, 2nd ed. (Clarendon, Oxford, 1978).

${ }^{13} \mathrm{~J}$. P. Biersack and D. Fink, in Ion Implantation into Semiconductors and Other Materials, edited by T. Namba (Plenum, New York, 1975), p. 211.

${ }^{14}$ M. Behar, J. P. Biersack, P. F. P. Fichtner, D. Fink, C. A. Olivieri, and F. C. Zawislak, Nucl. Instrum. Methods B 14, 173 (1986). 